\title{
Multistage Transmission Expansion Planning Using Local Branching Method
}

\section{Somphop Asadamongkol* and Bundhit Eua-arporn}

\author{
Department of Electrical Engineering, Faculty of Engineering, \\ Chulalongkorn University, Bangkok, 10330, Thailand \\ E-mail:somphop.as@student.chula.ac.th*,bundhit.e@chula.ac.th
}

\section{ABSTRACT}

This paper proposes a method for solving a large-scale multistage transmission expansion planning problem. The formulated problem is of a large-scale mixed integer linear programming which is difficult to handle by general mathematical optimization techniques. A disjunctive model for a single stage transmission expansion planning is extended for solving the multistage model. In addition, a local branching method is employed with an initial solution obtained from the single stage transmission expansion planning problem. The proposed method has been tested with IEEE 24-bus and a northeastern Thailand power system to show it is efficient to implement for actual power system planning.

\section{KEYWORDS}

disjunctive model, local branching method, mixed integer linear programming, multistage transmission expansion planning. 


\section{Introduction}

Electric power system is classified into generation system, transmission system and distribution system. A main function of the transmission system is to transfer electrical power from power plants to distribution systems, which eventually distribute power to customers. Consequently, when electricity demand is increased, the transmission system has to be reinforced. Transmission expansion planning (TEP) is a process for determining a minimum cost plan which ensures that the electricity demand can be served through a planning horizon. The TEP can be divided into two categories in view of planning horizon, i.e. single stage planning and multistage planning [1]. In the single stage planning, the planning horizon is considered as a single period of time. Therefore, all of transmission lines under the investment plan are assumed to be constructed at the same time, i.e. at the beginning of the considered period. Generally, the problem concerns only where to construct new lines and what types of lines to be constructed. Consequently, the single stage planning may not be appropriate for a long term TEP according to economical aspect, since it does not take into account the time value of the money. In case of the multistage, the planning horizon is divided into several stages, and each stage has its owned corresponding plan. The multistage planning problem concerns with questions about when and where to construct new transmission lines. The plan in the current stage depends on the plans in the previous stages. Therefore, the multistage planning is much more complicate than the single stage planning.

The TEP is a mixed integer programming (MIP) problem. There are three key groups of the methodologies developed to solve the TEP, i.e. mathematics, heuristics and metaheuristics.

The mathematical based methods [2] rigorously solve the problem by applying optimization techniques. In case of TEP, they are generally based on branch and bound algorithms. As long as the problem is convex, the methods can return a global optimal plan, however with high computational time, especially in case of large-scale problems. On the other hand, the heuristic based methods $[3,4,5]$ utilize some guidelines for searching solutions. For the TEP problem, they are usually derived from sensitivity indices regarding potential of candidates to alleviate the violation of operating limits. Even though the computational burden is low, the obtained solutions are usually trapped at local optima. The complexity of the heuristic methods may range from a simple greedy algorithm [6], to sophisticated methods, e.g. local branching [7]. The metaheuristic methods employ intelligent search techniques. The computation time is usually high and, in some cases, may be higher than the mathematical based methods. In fact, the metaheuristic methods are appropriate for nonconvex problems since they have mechanisms to escape the local optima. However, the solutions are not guaranteed to be a global optimum. Examples of the metaheuristic methods are genetic algorithm (GA) [8], tabu search [9], etc. From all the above methods, it should be noted that there is no best method which is suitable for all types of the TEP problems. Selecting the suitable method is mainly based on size of the problem as well as the model used in problem formulation.

A DC power flow is usually adopted in the TEP problem formulation. The decision variables are related to the selection of candidate branches into the plan. There are two kinds of decision variables, i.e. integer and binary variables. The integer variables represent the number of circuits of the candidates [10,11]. Therefore, the branch susceptance of each candidate is expressed in a function of the integer variable, and there exist the multiplication terms between the integer variables and the voltage angle variables in constraints corresponding to Kirchhoff's voltage law (KVL). These terms cause the problem to be a mixed integer nonlinear programming (MINLP). Some relaxed models have been used to reduce the computational burden of the formulated problem, e.g. transportation model [11] and hybrid model [2]. However some constraints are neglected in those models. Consequently, the obtained results are of the underinvestment plans. The binary variables represent the decision on selection of the candidate circuits [12, 13]. The disjunctive model proposed in [12] belongs to this kind of decision variables. The formulated problem is mixed integer linear programming (MILP), while the solution complies all constraints of the DC power flow.

Generally, the formulation of the multistage TEP is based on that of the single stage TEP. The derived multistage model will be inherited all properties and efficiencies from the original single stage model. Examples of the previous works can be found in [14] and [15]. For the formulation proposed in [14], the DC model is employed based on the integer decision variables. Consequently, there are multiplication terms between the decision variables and 
the voltage angle variables in the problem. As a result, the problem is a large-scale MINLP which is extremely complex. Since the problem is nonconvex, the GA is applied. However, the computational burden may be reasonably high.

In the case of the formulation presented in [15], the transportation model is applied with a constructive heuristic algorithm. Even though the formulated problem is MILP which is very simpler than MINLP, the KVL constraints are neglected in the formulation. Therefore, the obtained plan may be infeasible. Compared to other models, the obtained plan can lead to the underinvestment in practical planning activity.

In this paper, an extended version of disjunctive model is developed for the multistage TEP. It has an advantage over the DC model, since the formulated problem is an MILP which is easier to solve than the MINLP. In addition, the KVL constraints neglected in the transportation model are taken into account in the proposed model. However, for large-scale power systems, the problem is still difficult to handle by general mathematical optimization methods. Therefore, the local branching method [7] is employed to solve this problem. The advantage of the local branching method is that it is based on a local search technique. With the initial solution obtained from solving the consecutive single stage TEP, the optimal solution can be found with less computational burden.

As the results shown in [14] and [15], only the investment cost is taken into account in this paper. It should be noted that the objective function of the formulation in [14] apparently involves a value of lost load. However, the loss of load has no impact on the obtained investment plan.

\section{Problem Formulation}

\subsection{Single Stage Disjunctive Model}

The disjunctive model for the single stage TEP $[12,13]$ can be formulated as follows:

subject to

$$
\min \mathbf{c}^{\top} \mathbf{x}
$$

$$
\begin{aligned}
& \mathbf{A}_{\mathrm{g}}^{\top} \mathbf{p}_{\mathrm{g}}-\mathbf{A}_{\mathrm{be}}^{\top} \mathbf{p}_{\mathrm{be}}-\mathbf{A}_{\mathrm{bc}}^{\top} \mathbf{p}_{\mathrm{bc}}=\mathbf{p}_{\mathrm{d}} \\
& \mathbf{p}_{\mathrm{be}}-\mathbf{B}_{\mathrm{e}} \mathbf{A}_{\mathrm{be}} \boldsymbol{\theta}=\mathbf{0} \\
& -\mathbf{M}(\mathbf{1}-\mathbf{X}) \leq \mathbf{p}_{\mathrm{bc}}-\mathbf{B}_{\mathrm{c}} \mathbf{A}_{\mathrm{bc}} \boldsymbol{\theta} \leq \mathbf{M}(\mathbf{1}-\mathbf{X}) \\
& \mathbf{p}_{\mathrm{g}}^{\min } \leq \mathbf{p}_{\mathrm{g}} \leq \mathbf{p}_{\mathrm{g}}^{\max } \\
& -\mathbf{p}_{\mathrm{be}}^{\max } \leq \mathbf{p}_{\mathrm{be}} \leq \mathbf{p}_{\mathrm{be}}^{\max } \\
& -\mathbf{P}_{\mathrm{bc}}^{\max } \mathbf{X} \leq \mathbf{p}_{\mathrm{bc}} \leq \mathbf{P}_{\mathrm{bc}}^{\max } \mathbf{X}
\end{aligned}
$$

The variables and constant parameters in the formulation are described below.

\section{Variables}

$\mathbf{x} \in\{0,1\}^{n c}$ is a vector of binary variables which represent a decision on selection of corresponding circuits of the candidates into the considered plan,

$\mathbf{p}_{\mathrm{g}} \in \mathbb{R}^{n g}$ is a vector of power generation,

$\mathbf{p}_{\mathrm{be}} \in \mathbb{R}^{n e}$ and $\mathbf{p}_{\mathrm{bc}} \in \mathbb{R}^{n c}$ are vectors of power flow in the existing and candidate branches respectively, and

$\boldsymbol{\theta} \in \mathbb{R}^{n b}$ is a vector of voltage angle. 


\section{Constant parameters}

$\mathbf{c} \in \mathbb{R}^{n c}$ is a vector representing construction cost per circuit of the candidate branches, $\mathbf{A}_{\mathrm{g}} \in \mathbb{R}^{n \times \times n b}$ is a generator-bus incidence matrix,

$\mathbf{A}_{\mathrm{be}} \in \mathbb{R}^{n e \times n b}$ and $\mathbf{A}_{\mathrm{bc}} \in \mathbb{R}^{n c \times n b}$ are branch-bus incidence matrices for existing and candidate branches respectively,

$\mathbf{p}_{\mathrm{d}} \in \mathbb{R}^{n b}$ is a vector of power demand,

$\mathbf{B}_{\mathbf{e}} \in \mathbb{R}^{\text {nexne }}$ and $\mathbf{B}_{\mathrm{c}} \in \mathbb{R}^{n c \times n c}$ are diagonal matrices of which the elements representing susceptance of existing and candidate branches respectively,

$\mathbf{M} \in \mathbb{R}^{n c \times n c}$ is a diagonal matrix of which the elements representing disjunctive parameters which can be calculated by the method proposed in [13],

$\mathbf{p}_{\mathrm{g}}^{\min }$ and $\mathbf{p}_{\mathrm{g}}^{\max } \in \mathbb{R}^{n g}$ are vectors of the minimum and maximum limits of power generation,

$\mathbf{p}_{\mathrm{be}}^{\max } \in \mathbb{R}^{n e}$ is a vector of the maximum limits of power flow in existing branches,

$\mathbf{P}_{\mathrm{bc}}^{\max } \in \mathbb{R}^{n c \times n c}$ is a diagonal matrix of which the elements representing maximum limits of power flow in candidate branches,

$n g, n b$, ne, and $n c$ are the number of generators, the number of buses, the number of existing branches, and the number of candidate branches, respectively.

It is clearly seen that the objective function is the investment cost function shown by Eq. (1). All the constraints comply with the DC power flow model as well as operation limits. Considering the constraints (4) and (7), one can express the constraints corresponding to the circuit $l$ which directly connects from bus $f$ to bus $t$ as follows:

$$
\begin{aligned}
& \left|p_{l}-B_{l}\left(\theta_{f}-\theta_{t}\right)\right| \leq M_{l}\left(1-x_{l}\right) \\
& \left|p_{l}\right| \leq p_{l}^{\max } x_{l}
\end{aligned}
$$

It should be noticed that if the candidate branch $l$ is selected for the plan, i.e. $x_{l}=1$, the constraint (8) will comply with the KVL. On the other hand, if the candidate branch $l$ is not selected, i.e. $x_{l}=0, p$, will be zero by the constraint (9). In addition, $\theta_{f}$ and $\theta_{t}$ are not controlled by the constraint (8) due to the large value of $M_{l}$.

\subsection{Multistage Disjunctive Model}

In general, the multistage TEP can be considered as a sequence of the single stage TEP. The planning horizon and the investment plan corresponding to each stage of the multistage TEP can be demonstrated in Figure 1. In this figure, it is assumed that the planning period is nine years, which is divided into three stages. The plan is carried out at the beginning of each stage in order that the increased demand in the corresponding stage can be served.

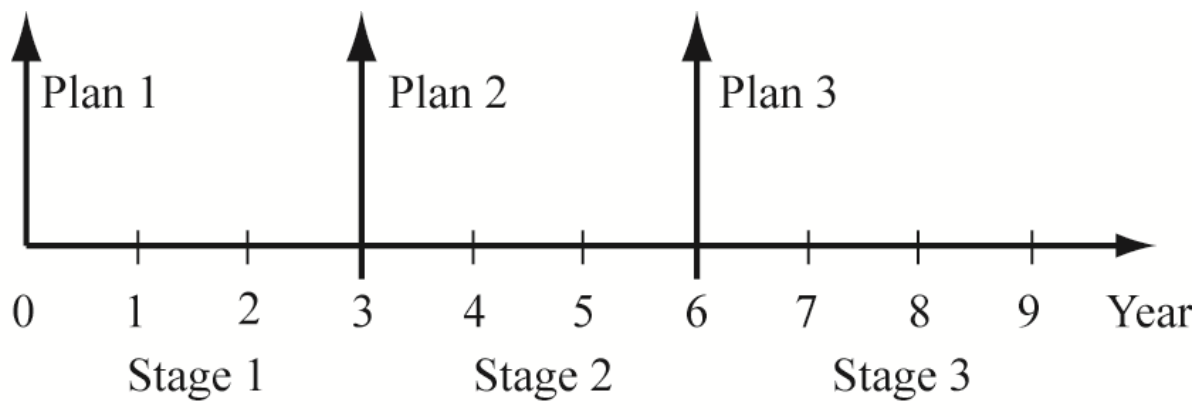

Figure 1

Planning horizon of Multistage TEP

The plans obtained from the previous stages have to be perceived at the current stage. Therefore, additional constraints should be introduced into the single stage disjunctive model, and some existing constraints in the single stage disjunctive model should be 
modified. The proposed model for multistage TEP comprising $n s$ stages can be expressed as follows:

$$
\min \left\{\sum_{t=1}^{n s} \delta^{(t)} \mathbf{c}^{\top} \mathbf{x}^{(t)}\right\}
$$

subject to

$$
\begin{aligned}
& \mathbf{A}_{\mathbf{g}}^{\top} \mathbf{p}_{\mathbf{g}}^{(t)}-\mathbf{A}_{\mathbf{b e}}^{\top} \mathbf{p}_{\mathbf{b e}}^{(t)}-\mathbf{A}_{\mathbf{b c}}^{\top} \mathbf{p}_{\mathbf{b c}}^{(t)}=\mathbf{p}_{\mathbf{d}}^{(t)} \\
& \mathbf{p}_{\mathbf{b e}}^{(t)}-\mathbf{B}_{\mathbf{e}} \mathbf{A}_{\mathbf{b e}} \boldsymbol{\theta}^{(t)}=\mathbf{0} \\
& -\mathbf{M}\left(\mathbf{1}-\sum_{h=1}^{t} \mathbf{x}^{(h)}\right) \leq \mathbf{p}_{\mathbf{b c}}^{(t)}-\mathbf{B}_{\mathbf{c}} \mathbf{A}_{\mathbf{b c}} \boldsymbol{\theta}^{(t)} \leq \mathbf{M}\left(\mathbf{1}-\sum_{h=1}^{t} \mathbf{x}^{(h)}\right) \\
& \mathbf{p}_{\mathbf{g}}^{\min ,(t)} \leq \mathbf{p}_{\mathbf{g}}^{(t)} \leq \mathbf{p}_{\mathbf{g}}^{\max ,(t)} \\
& -\mathbf{p}_{\mathbf{b e}}^{\max } \leq \mathbf{p}_{\mathbf{b e}}^{(t)} \leq \mathbf{p}_{\mathbf{b e}}^{\max } \\
& -\mathbf{P}_{\mathbf{b c}}^{\max }\left(\sum_{h=1}^{t} \mathbf{x}^{(h)}\right) \leq \mathbf{p}_{\mathbf{b c}}^{(t)} \leq \mathbf{P}_{\mathbf{b c}}^{\max }\left(\sum_{h=1}^{t} \mathbf{x}^{(h)}\right)
\end{aligned}
$$

The constraints (11)-(16) are stated for $t=1, \ldots, n s$.

$$
\sum_{h=1}^{n s} \mathbf{x}^{(h)} \leq 1
$$

where $\delta^{(t)}$ is a discount factor for stage $t$ which can be expressed as

$$
\delta^{(t)}=\left(\frac{1}{1+r}\right)^{(t-1) n y}=(1-d)^{(t-1) n y}
$$

$r$ is an interest rate (\% per year), $d$ is a discount rate (\% per year), and $n y$ is the number of years for each stage.

It should be emphasized that there are three points which are different from the single stage disjunctive model [12] as described below.

- All variables in the single stage disjunctive model are extended for every other stage. In addition, the power demand and generation capacity should be varied according to load forecast and generation expansion plan,

- The constraints (13) and (16) are modified from the constraints (4) and (7) in order that the current stage will recognize the plans in the previous stages, and

- The additional constraint (17) ensures that circuits of the candidate branches can be selected into the plan for only one stage.

\section{Local Branching Method}

Local branching method is proposed by M. Fischetti and A. Lodi [7]. It is a heuristic method for solving an MILP. Actually, the local branching method is a local search procedure integrated with diversification process. It is shown in [7] that the local branching method can find a global minimum within computational time less than the mathematical based methods in many cases of large-scale MILPs. Moreover, in cases of complicated MILPs of which the solutions cannot find by both the mathematic based methods and the local branching method in a specified time, the local branching method may provide the feasible solutions better than the ones obtained from the mathematical optimization methods. Details of the local branching method have been summarized in the following two subsections, whereas more details can be found in [7]. 


\subsection{Local Search}

The main concept of the local branching method is to perform a local search in a neighborhood of a reference solution. With a specified reference solution $\overline{\mathbf{x}}$, a k-OPT neighborhood $\mathcal{N}(\overline{\mathbf{x}}, k)$ is defined as follows:

$$
\mathcal{N}(\overline{\mathbf{x}}, k)=\{\mathbf{x}, \Delta(\mathbf{x}, \overline{\mathbf{x}}) \leq k\}
$$

where $k$ is a constant related to size of the neighborhood, and $\Delta(\mathbf{x}, \overline{\mathbf{x}}) X_{1}, \ldots, X_{n}$ is Hamming distance defined as

$$
\Delta(\mathbf{x}, \overline{\mathbf{x}})=\sum_{j \in \bar{S}}\left(1-x_{j}\right)+\sum_{j \in \mathcal{B} \backslash \bar{S}} x_{j}
$$

$\mathcal{B}$ is an index set of the binary variable $\mathbf{x}$, and

$$
\bar{S}=\left\{j \in \mathcal{B}, \quad \bar{x}_{j}=1\right\}
$$

The local search is performed in $\mathcal{F} \cap \mathcal{N}(\overline{\mathbf{x}}, k)$ where $\mathcal{F}$ is a feasible set of solutions of the original problem. It should be noted that the local branching method utilizes a general MILP solver to perform the local search by appending the following constraint into the original problem.

$$
\Delta(\mathbf{x}, \overline{\mathbf{x}})=\sum_{j \in \bar{S}}\left(1-x_{j}\right)+\sum_{j \in \mathcal{B} \backslash \bar{S}} x_{j} \leq k
$$

The process of the local branching method is initialized with a starting solution $\overline{\mathbf{x}}_{1}$, which can be established from the first feasible solution found by the MILP solver. With the current feasible set of solutions $\mathcal{F}$, the local search is performed in $\mathcal{F} \cap \mathcal{N}\left(\overline{\mathbf{x}}_{1}, k\right)$ within a given time limit. The value of objective function at $\overline{\mathbf{x}}_{1}$ is used as an upper bound for an MILP solver. After the solver is terminated, the following situations may occur.

- An optimal solution is found. This solution is an optimal solution in $\mathcal{F} \cap \mathcal{N}\left(\overline{\mathbf{x}}_{1}, k\right)$, however it may not be a global optimal of the original MILP problem. Therefore, it will be assigned to be the new incumbent $\overline{\mathbf{x}}_{2}$. After that the current feasible set is updated to $\mathcal{F}=\mathcal{F} \backslash \mathcal{N}\left(\overline{\mathbf{x}}_{1}, k\right)$ and the local search is performed again in $\mathcal{F} \cap \mathcal{N}\left(\overline{\mathbf{x}}_{2}, k\right)$. The value of objective function at the new incumbent is subsequently used as the upper bound for MILP solver.

- No optimal solution is found within the specified time limit, however the MILP solver terminated with a feasible solution. In this case the feasible solution will be assigned to be the new incumbent $\overline{\mathbf{x}}_{2}$, and the current feasible set is updated to $\mathcal{F}=\mathcal{F} \backslash\left\{\overline{\mathbf{x}}^{1}\right\}$. The local search is performed again in $\mathcal{F} \cap \mathcal{N}\left(\overline{\mathbf{x}}_{2}, k\right)$, and the value of objective function at the new incumbent is used as the upper bound for MILP solver.

- The MILP solver cannot find any feasible solution within the time limit. To cope with this situation, the current neighborhood is modified to be a smaller one, i.e. $\mathcal{N}\left(\overline{\mathbf{x}}^{1},\lceil k / 2\rceil\right)$. After performing the local search in $\mathcal{F} \cap \mathcal{N}\left(\overline{\mathbf{x}}^{1},\lceil k / 2\rceil\right)$, if the feasible solution cannot be found, strong diversification process, which will be shown in the following subsection, will be applied.

- The MILP solver is terminated due to an infeasible problem. The diversification process will be applied to find a new incumbent solution. Then the local search is performed again. It should be noted that the soft diversification process is employed at first when this situation arises. After performing the local search, if this situation occurs again, the strong diversification process will be applied. 
The local search is continuously executed in this manner until the total time limit is reached. In addition, the best solution found during the process will be recorded, and finally used as the solution.

\subsection{Diversification}

When the problem is not feasible, the size of the current neighborhood will be enlarged by $\lceil k / 2\rceil$, and the local search is performed to find the optimal solution in the extended neighborhood. The obtained solution will be used as the new incumbent for the subsequent process of the local search described in the previous subsection. This concept is called soft diversification. If the problem is still infeasible after applying the soft diversification, the strong diversification will be employed by enlarging the current neighborhood by $\lceil k / 2\rceil$, and performing the local search by MILP solver. However, in the case of the strong diversification, the solver will find the first feasible solution instead of the optimal solution which is found in the soft diversification case. Then the solution will be used as the new incumbent for the next process of the local search in the previous subsection. The strong diversification will be also applied in the situation that the solver cannot find any feasible solution in the current neighborhood within the specified time limit.

\subsection{Local Branching Method for Multistage TEP}

From the previous subsections, one can see that the main algorithm of the local branching method is based on the local search. However, the searching procedure is more sophisticated than general local search procedures. It should be remarked that an initial solution will affect the quality of an optimal solution found in the final calculation. This feature may contribute to the advantage in some applications for which good initial solution can be found easily, e.g. the multistage TEP. Since the computational time for solving the consecutive single stage TEP is much less than the time for solving the multistage TEP. Therefore, one can use the solution of the consecutive single stage TEP as the initial solution of the multistage TEP. The plans in the previous stages have to be updated to the existing branches before solving the single stage TEP for the current stage. On the assumption that the optimal plan of the multistage TEP is close to the plan of the consecutive single stage TEP, the local branching method should find good quality solutions for large-scale problems within acceptable computational time.

\section{Results and Discussion}

In this section, the multistage TEP is formulated by using the proposed disjunctive model and solved by the local branching method. The solution obtained by the consecutive single stage TEP is used as the initial solution of the multistage TEP. The test systems are composed of IEEE 24-bus and northeastern Thailand systems. The length of the planning period for both systems is nine years. It is assumed that the planning period can be divided into three stages, of which each interval spans three years. The interest rate is set at $10 \%$ per year. The structure of planning periods and stages for both multistage TEP problems are similar to one demonstrated in Figure 1. TOMLAB/CPLEX is employed as the MILP solver.

\subsection{IEEE-24 Bus System}

The system consists of 24 buses, 38 existing branches and 41 candidates. The maximum number of circuits on each candidate is three. In this test system, the data for the single stage TEP in [16] is applied for the multistage TEP. It is assumed that the power demand and the generation capacity monotonously increase through the planning period. Therefore, the plan established at the beginning of each stage must be able to serve the demand at the end of stage. For this reason, the power demand and the generation capacities at year 3, 6 and 9 will be used as the representative values of the first, second, and third stages respectively.

In the first stage, the power demand and the installed generation capacity are 8,550 MW and 10,215 MW respectively. These values are defined according to the data for the single stage TEP presented in [16]. For the next two stages, the demand is assumed to grow by $8 \%$ per year. Subsequently, to obtain the demand of the second and third stages, the demand of the first stage at every bus is multiplied by $1.26\left(1.08^{3}\right)$ 
and $1.59\left(1.08^{6}\right)$, respectively. In addition, the installed generation capacities at the end of the second and third stages increase by $25 \%$ and $50 \%$ from the value of the first stage respectively.

In the beginning, three consecutive single stage TEP is performed. The net present value of the investment cost is $594.01 \times 10^{4}$ US $\$$. This value is used as an upper bound of MILP solver for the local branching method. In addition, the obtained plan is also used as the initial solution.

The parameters for the local branching method applied to the IEEE-24 bus test system are set as follows: the size of neighborhood $k$ is 5 , the time limit for searching in the current neighborhood is $300 \mathrm{sec}$., and the total time limit is $3600 \mathrm{sec}$. The solutions found during the process of the local branching method for the multistage TEP are illustrated in Figure 2. The result with comparison to the consecutive single stage TEP is shown in Table 1.

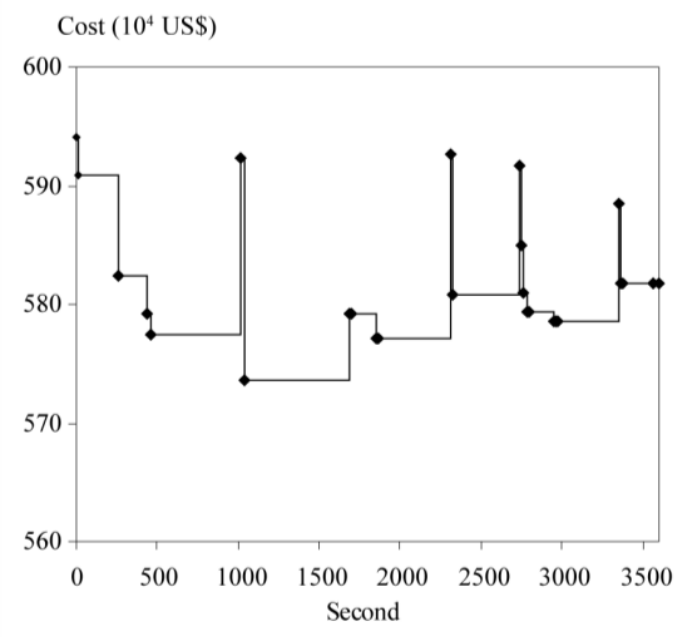

\begin{tabular}{lccccc}
\hline \multirow{2}{*}{ Results } & \multicolumn{2}{c}{ Multistage TEP } & & \multicolumn{2}{c}{ Single stage TEP } \\
\cline { 2 - 3 } \cline { 5 - 6 } & Bus & Number & & Bus & Number \\
\hline First stage & $6-10$ & 1 & & $6-10$ & 1 \\
& $7-8$ & 2 & & $7-8$ & 2 \\
& $10-12$ & 1 & & $10-12$ & 1 \\
& $14-16$ & 1 & & $14-16$ & 1 \\
\hline Second stage & $1-5$ & 1 & & $1-5$ & 1 \\
& $3-24$ & 1 & & $6-10$ & 1 \\
& $6-10$ & 1 & & $7-8$ & 1 \\
& $7-8$ & 1 & & $9-11$ & 1 \\
& $12-13$ & 1 & & $11-13$ & 1 \\
& $15-24$ & 1 & & $12-13$ & 1 \\
& $16-17$ & 1 & & $20-23$ & 1 \\
& $20-23$ & 1 & & $1-5$ & 1 \\
& $2-4$ & 1 & & $3-24$ & 1 \\
& $3-24$ & 1 & & $4-9$ & 1 \\
& $9-11$ & 1 & & $10-11$ & 1 \\
& $10-12$ & 1 & & $14-23$ & 2 \\
& $14-23$ & 1 & & $15-24$ & 1 \\
& $15-21$ & 1 & & $16-17$ & 1 \\
\hline
\end{tabular}

Net Present

Value (10 4 US $\$$ )
573.63
Figure 2

Solutions found during the process for IEEE24 bus system

Table 1

Comparison of results for IEEE 24-bus system 


\subsection{Northeastern Thailand System}

The system consists of 75 buses, 153 existing branches and 129 candidates. It is modified from an actual system. The maximum number of circuits on each candidate is allowed at three. The active power demand is $4,634.4 \mathrm{MW}$ and the installed generation capacity is $6,824.0 \mathrm{MW}$ at the end of the first stage. Detailed system information is shown in Appendix.

The demand is assumed to grow by $5 \%$ per year. Therefore, the demand at the end of the second and third stages are calculated by multiplying $1.16\left(1.05^{3}\right)$ and $1.34\left(1.05^{6}\right)$ to the value of the first stage respectively. The installed generation capacities increase from the value of the first stage by $20 \%$ for the second stage and $35 \%$ for the third stage.

The consecutive single stage TEP is employed to find the initial solution for the local branching method. The value of the upper bound is $32,355.1 \times 10^{3}$ US $\$$.

The local branching method applied to this test system is performed with the following parameters: the size of neighborhood $k$ is 10 , the time limit for searching in the current neighborhood is $500 \mathrm{sec}$. , and the total time limit is $7200 \mathrm{sec}$. The solutions found during the process of the local branching method are illustrated in Figure 3. Comparison of the multistage TEP to the consecutive single stage TEP is shown in Table 2. In this test system, some candidates which connect between same terminals have different capacities. Therefore, the expanded capacities between two terminal buses instead of the number of circuits are indicated in the table.

\subsection{Discussion}

The benefit of multistage TEP of medium-scale and large-scale power systems has been clearly shown with the application of the local branching method. The investment cost can be reduced by $3.4 \%$ for the IEEE 24-bus system, and $3.5 \%$ for the northeastern Thailand system. From the results, it should be noticed that the more investment in the second stage can be reduce the expansion in the third stage, and results in the lower investment cost in the overall planning horizon. In view of the computational efficiency, one can see that the best solution can be found within acceptable time periods, i.e. $1033 \mathrm{sec}$. for the IEEE 24-bus system and $496 \mathrm{sec}$. for the northeastern Thailand system. It should be also noted that the shorter time in case of the northeastern Thailand system, which is the larger system, can be well explained. Since the local branching method does not guarantee the global optimality of the solution, and one cannot ensure that the solution quality in case of the northeastern Thailand system is comparable to the solution quality in case of the IEEE 24-bus system.

The parameters of the local branching method are critical issues which can affect the overall performance. The size of neighborhood $k$ and the time limit for searching the solution in the current neighborhood should be selected accordingly. In case of the larger neighborhood, the better solution might be found within execution time longer than the case of smaller one. For the large-scale systems, the solution quality and the computational time should be compromised. 
Figure 3

Solutions found during the process for

northeastern Thailand system
Cost $\left(10^{3}\right.$ US\$)

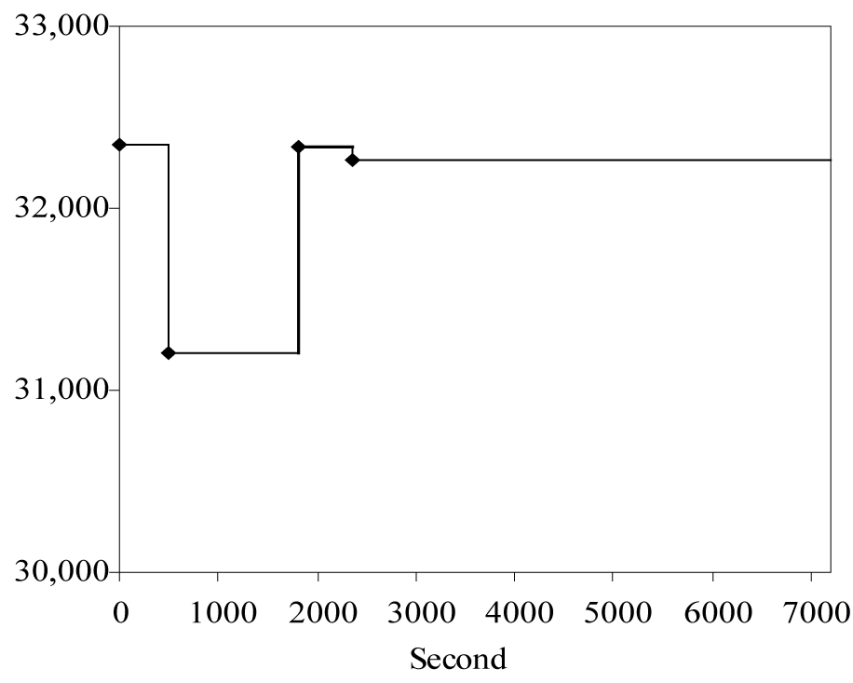

Table 2

Comparison of results for northeastern

Thailand system

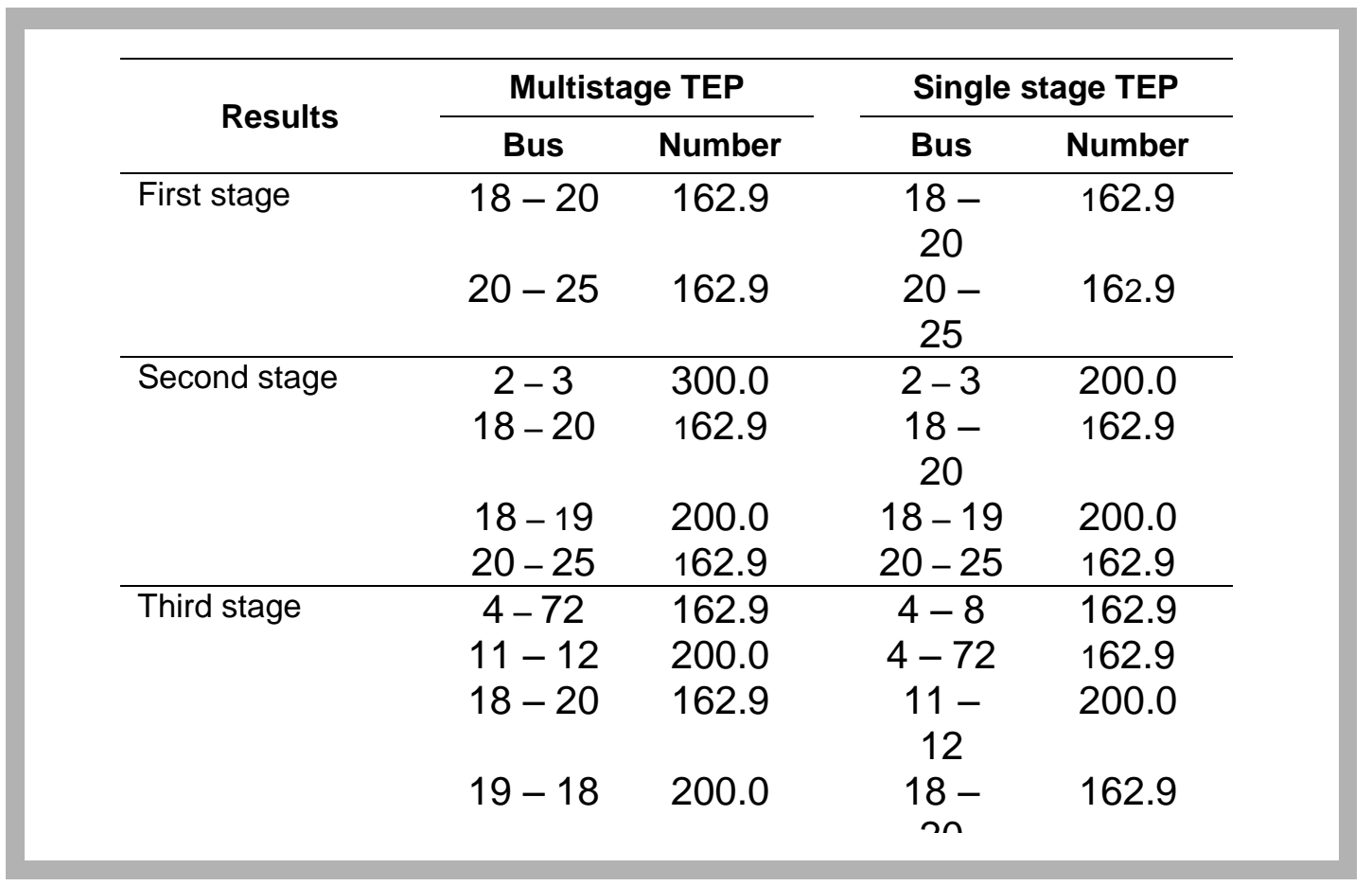

\section{Conclusion}

A multistage disjunctive model is proposed in this paper with further development from the model proposed for the single stage TEP. The formulated problem is an MILP which can be handled by various algorithms and solvers. This is an advantage of the disjunctive model over other models. The proposed methodology focuses on the case of medium-scale and large-scale power systems. Therefore, the local branching method which is appropriate for large-scale MILP is applied to solve the problems. The results are compared with the ones obtained from the single stage TEP calculation. It clearly shows the benefit of the multistage TEP from economical point of view. For a large-scale power system, a long term investment plan can be obtained within acceptable computational time. Therefore, the proposed model accompanying with the solving procedure is applicable for actual transmission planning activities. 


\section{Appendix}

Bus and branch data of the modified northeastern Thailand test system are listed in Tables 3 and 4. The active, reactive, and apparent power are expressed in MW, MVAr, and MVA, respectively. The branch parameters, i.e. $r_{i j}, x_{i j}$ and $b_{i j}$, are expressed in per unit based on 100 MVA. The cost is expressed in thousand US $\$$. The $n^{\circ}$ and $n^{\max }$ are the number of circuits of existing branches and the maximum number of circuits of candidate branches respectively.

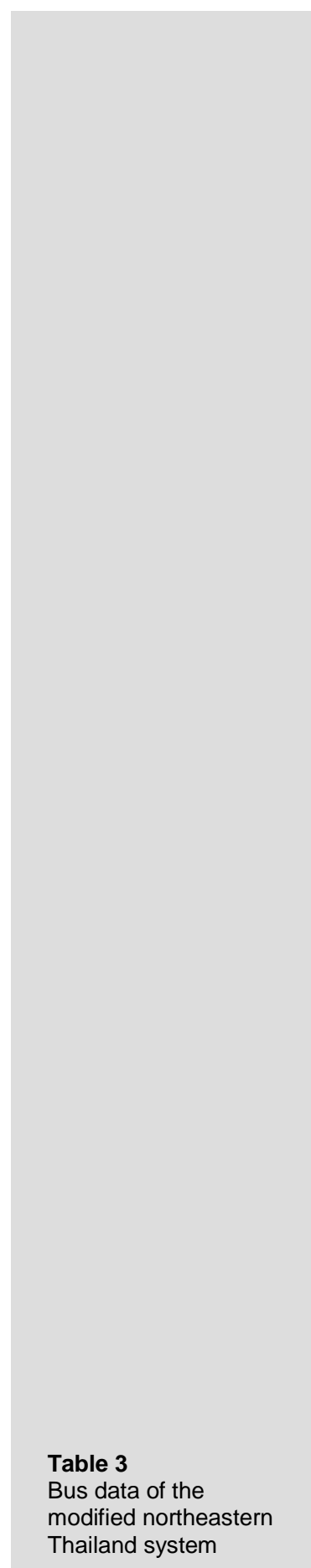

\begin{tabular}{|c|c|c|c|c|c|c|c|}
\hline Bus & Type & $p_{1}$ & $q_{1}$ & $p_{g} \max$ & $p_{g} \min$ & $q_{g} \max$ & $q_{g} \min$ \\
\hline 1 & $P Q$ & 91.8 & 50.8 & - & - & - & - \\
\hline 2 & PV & 32.4 & 20.1 & 1350.0 & 0.0 & 711.0 & -356.0 \\
\hline 3 & $P Q$ & - & - & - & - & - & - \\
\hline 4 & PV & 136.4 & 75.5 & 54.0 & 0.0 & 28.0 & -14.0 \\
\hline 5 & $P Q$ & 55.3 & 30.6 & - & - & - & - \\
\hline 6 & $P Q$ & 114.2 & 63.2 & - & - & - & - \\
\hline 7 & $P Q$ & 5.3 & 2.9 & - & - & - & - \\
\hline 8 & $P Q$ & 48.3 & 26.7 & - & - & - & - \\
\hline 9 & $P Q$ & 29.2 & 16.2 & - & - & - & - \\
\hline 10 & $P Q$ & 94.0 & 52.0 & - & - & - & - \\
\hline 11 & $P Q$ & 160.3 & 88.7 & - & - & - & - \\
\hline 12 & PV & - & - & 900.0 & 0.0 & 474.0 & -237.0 \\
\hline 13 & PV & 0.9 & 0.6 & 90.0 & 0.0 & 47.0 & -24.0 \\
\hline 14 & PV & 63.4 & 35.1 & 90.0 & 0.0 & 47.0 & -24.0 \\
\hline 15 & PV & 1.8 & 1.1 & 234.0 & 0.0 & 123.0 & -62.0 \\
\hline 16 & $P Q$ & 79.1 & 43.7 & - & - & - & - \\
\hline 17 & $P Q$ & 54.2 & 30.0 & - & - & - & - \\
\hline 18 & $P Q$ & 241.4 & 133.6 & - & - & - & - \\
\hline 19 & $P Q$ & - & - & - & - & - & - \\
\hline 20 & $P Q$ & 83.6 & 46.3 & - & - & - & - \\
\hline 21 & PV & - & - & 900.0 & 0.0 & 474.0 & -237.0 \\
\hline 22 & $P Q$ & 135.7 & 75.1 & - & - & - & - \\
\hline 23 & SL & 0.9 & 0.6 & 1800.0 & 0.0 & 948.0 & -474.0 \\
\hline 24 & $P Q$ & 99.3 & 54.9 & - & - & - & - \\
\hline 25 & $P Q$ & 136.2 & 75.3 & - & - & - & - \\
\hline 26 & $P Q$ & 36.6 & 21.9 & - & - & - & - \\
\hline 27 & $P Q$ & 73.2 & 40.5 & - & - & - & - \\
\hline 28 & $P Q$ & 170.3 & 94.2 & - & - & - & - \\
\hline 29 & $P Q$ & - & - & - & - & - & - \\
\hline 30 & $P Q$ & 95.2 & 52.7 & - & - & - & - \\
\hline 31 & $P Q$ & 572.2 & 316.6 & - & - & - & - \\
\hline 32 & $P Q$ & - & - & - & - & - & - \\
\hline 33 & PV & - & - & 108.0 & 0.0 & 57.0 & -29.0 \\
\hline 34 & PV & 1.8 & 1.1 & 171.0 & 0.0 & 90.0 & -45.0 \\
\hline 35 & PV & 0.9 & 0.6 & 11.0 & 0.0 & 60. & -3.0 \\
\hline
\end{tabular}




\begin{tabular}{|c|c|c|c|c|c|c|c|}
\hline Bus & Type & $p_{1}$ & $q_{1}$ & $p_{g} \max$ & $p_{g} \min$ & $q_{g} \max$ & $q_{g} \min$ \\
\hline 36 & $P Q$ & 9.2 & 5.1 & - & - & - & - \\
\hline 37 & $P Q$ & 63.1 & 64.9 & - & - & - & - \\
\hline 38 & $P Q$ & 51.0 & 28.2 & - & - & - & - \\
\hline 39 & $P Q$ & 168.3 & 93.1 & - & - & - & - \\
\hline 40 & PV & 71.7 & 39.6 & 216.0 & 0.0 & 6.0 & -3.0 \\
\hline 41 & PV & 1.8 & 1.1 & 126.0 & 0.0 & 66.0 & -33.0 \\
\hline 42 & $P Q$ & - & - & - & - & - & - \\
\hline 43 & $P Q$ & 26.8 & 14.8 & - & - & - & - \\
\hline 44 & $P Q$ & 135.1 & 74.8 & - & - & - & - \\
\hline 45 & $P Q$ & 124.6 & 68.9 & - & - & - & - \\
\hline 46 & $P Q$ & 36.9 & 20.4 & - & - & - & - \\
\hline 47 & $P Q$ & 53.2 & 29.3 & - & - & - & - \\
\hline 48 & $P Q$ & - & - & - & - & - & - \\
\hline 49 & $P Q$ & 57.5 & 31.8 & - & - & - & - \\
\hline 50 & $P Q$ & 92.7 & 51.3 & - & - & - & - \\
\hline 51 & $P Q$ & - & - & - & - & - & - \\
\hline 52 & $P Q$ & - & - & - & - & - & - \\
\hline 53 & $P Q$ & 68.1 & 37.7 & - & - & - & - \\
\hline 54 & $P Q$ & - & - & - & - & - & - \\
\hline 55 & $P Q$ & - & - & - & - & - & - \\
\hline 56 & $P Q$ & 32.9 & 18.2 & - & - & - & - \\
\hline 57 & $P Q$ & 138.9 & 76.9 & - & - & - & - \\
\hline 58 & PV & 60.4 & 33.4 & 216.0 & 0.0 & 114.0 & -57.0 \\
\hline 59 & PV & 78.4 & 43.4 & 72.0 & 0.0 & 38.0 & -19.0 \\
\hline 60 & $P Q$ & 64.1 & 35.5 & - & - & - & - \\
\hline 61 & $P Q$ & 41.6 & 23.0 & - & - & - & - \\
\hline 62 & $P Q$ & - & - & - & - & - & - \\
\hline 63 & $P Q$ & - & - & - & - & - & - \\
\hline 64 & $P Q$ & - & - & - & - & - & - \\
\hline 65 & $P Q$ & 32.2 & 17.8 & - & - & - & - \\
\hline 66 & PV & 1.8 & 1.1 & 432.0 & 0.0 & 228.0 & -114.0 \\
\hline 67 & $\mathrm{PV}$ & 0.9 & 0.6 & 54.0 & 0.0 & 28.0 & -14.0 \\
\hline 68 & $P Q$ & 57.0 & 31.5 & - & - & - & - \\
\hline 69 & $P Q$ & 221.6 & 112.6 & - & - & - & - \\
\hline 70 & $P Q$ & - & - & - & - & - & - \\
\hline 71 & $P Q$ & 108.5 & 60.1 & - & - & - & - \\
\hline 72 & $P Q$ & 146.7 & 81.2 & - & - & - & - \\
\hline 73 & $P Q$ & - & - & - & - & - & - \\
\hline 74 & $P Q$ & 72.5 & 40.1 & - & - & - & - \\
\hline 75 & $P Q$ & - & - & - & - & - & - \\
\hline
\end{tabular}

Table 3

Bus data of the modified northeastern

Thailand system

(contimued) 


\begin{tabular}{|c|c|c|c|c|c|c|c|c|c|}
\hline & From & To & $r_{i j}$ & $x_{i j}$ & $b_{i j}$ & $S_{i f}^{\max }$ & cost & $n^{\circ}$ & $n^{\max }$ \\
\hline & 1 & 10 & 0.0662 & 0.1404 & 0.0171 & 96.4 & - & 1 & - \\
\hline & 1 & 10 & 0.0289 & 0.1272 & 0.0191 & 162.9 & 4,111 & - & 3 \\
\hline & 1 & 45 & 0.0580 & 0.2550 & 0.0383 & 162.9 & 7,529 & 1 & 3 \\
\hline & 1 & 56 & 0.0298 & 0.1309 & 0.0196 & 162.9 & 4,210 & - & 3 \\
\hline & 1 & 61 & 0.0008 & 0.0033 & 0.0005 & 162.9 & 804 & 2 & 3 \\
\hline & 2 & 3 & 0.0000 & 0.0621 & 0.0000 & 200.0 & - & 1 & - \\
\hline & 2 & 3 & 0.0000 & 0.0650 & 0.0000 & 200.0 & 3,132 & 1 & 3 \\
\hline & 2 & 3 & 0.0000 & 0.0550 & 0.0000 & 300.0 & 3,767 & - & 3 \\
\hline & 2 & 12 & 0.0129 & 0.0931 & 0.2044 & 429.4 & 14,584 & - & 3 \\
\hline & 2 & 19 & 0.0034 & 0.0244 & 0.0535 & 429.4 & 4,790 & - & 3 \\
\hline & 2 & 21 & 0.0015 & 0.0154 & 0.0628 & 858.9 & - & 2 & - \\
\hline & 2 & 21 & 0.0029 & 0.0211 & 0.0464 & 429.4 & 4,328 & - & 3 \\
\hline & 2 & 51 & 0.0129 & 0.0930 & 0.2043 & 429.4 & 14,581 & - & 3 \\
\hline & 2 & 52 & 0.0113 & 0.0812 & 0.1783 & 429.4 & 12,894 & - & 3 \\
\hline & 3 & 4 & 0.0019 & 0.0124 & 0.0032 & 325.9 & - & 2 & - \\
\hline & 3 & 4 & 0.0037 & 0.0163 & 0.0024 & 162.9 & 1,152 & - & 3 \\
\hline & 3 & 18 & 0.0213 & 0.0934 & 0.0140 & 162.9 & 3,209 & - & 3 \\
\hline & 3 & 20 & 0.0223 & 0.0980 & 0.0147 & 162.9 & 3,331 & - & 3 \\
\hline & 3 & 67 & 0.0117 & 0.0515 & 0.0077 & 162.9 & 2,091 & - & 3 \\
\hline & 4 & 18 & 0.0298 & 0.0836 & 0.0117 & 119.5 & - & 2 & - \\
\hline & 4 & 18 & 0.0184 & 0.0811 & 0.0122 & 162.9 & 2,880 & - & 3 \\
\hline & 4 & 20 & 0.0205 & 0.0901 & 0.0135 & 162.9 & 3,120 & - & 3 \\
\hline & 4 & 67 & 0.0623 & 0.0782 & 0.0083 & 67.1 & - & 1 & - \\
\hline & 4 & 67 & 0.0151 & 0.0663 & 0.0099 & 162.9 & 2,486 & - & 3 \\
\hline & 4 & 71 & 0.0991 & 0.2813 & 0.0387 & 119.5 & - & 2 & - \\
\hline & 4 & 71 & 0.0615 & 0.2705 & 0.0405 & 162.9 & 7,936 & - & 3 \\
\hline & 4 & 72 & 0.0832 & 0.2339 & 0.0328 & 325.9 & - & 2 & - \\
\hline & 4 & 72 & 0.0516 & 0.2269 & 0.0340 & 162.9 & 6,773 & - & 3 \\
\hline & 5 & 26 & 0.2398 & 0.3017 & 0.0323 & 67.1 & - & 1 & - \\
\hline & 5 & 26 & 0.0583 & 0.2561 & 0.0384 & 162.9 & 7,552 & - & 3 \\
\hline & 5 & 68 & 0.0409 & 0.1799 & 0.0270 & 162.9 & 5,519 & 1 & 3 \\
\hline & 5 & 74 & 0.0495 & 0.1390 & 0.0195 & 119.5 & - & 2 & - \\
\hline & 5 & 74 & 0.0307 & 0.1348 & 0.0202 & 162.9 & 4,315 & - & 3 \\
\hline & 6 & 11 & 0.0376 & 0.1653 & 0.0248 & 162.9 & 5,129 & 1 & 3 \\
\hline & 7 & 38 & 0.0412 & 0.1212 & 0.0154 & 119.5 & - & 1 & - \\
\hline & 7 & 38 & 0.0255 & 0.1121 & 0.0168 & 162.9 & 3,708 & - & 3 \\
\hline & 8 & 18 & 0.0399 & 0.1119 & 0.0157 & 119.5 & - & 1 & - \\
\hline & 8 & 18 & 0.0247 & 0.1085 & 0.0163 & 162.9 & 3,612 & - & 3 \\
\hline \multirow{4}{*}{$\begin{array}{l}\text { Table } 4 \\
\text { Branch data of the } \\
\text { modified northeastern } \\
\text { Thailand system }\end{array}$} & 8 & 20 & 0.0281 & 0.1234 & 0.0185 & 162.9 & 4,010 & - & 3 \\
\hline & 8 & 22 & 0.0975 & 0.2742 & 0.0385 & 119.5 & - & 1 & - \\
\hline & 8 & 22 & 0.0606 & 0.2662 & 0.0399 & 162.9 & 7,821 & - & 3 \\
\hline & 8 & 46 & 0.0235 & 0.1031 & 0.0155 & 162.9 & 3,468 & - & 3 \\
\hline
\end{tabular}




\begin{tabular}{|c|c|c|c|c|c|c|c|c|}
\hline From & To & $r_{i j}$ & $x_{i j}$ & $b_{i j}$ & $S_{i f}^{\max }$ & cost & $n^{\circ}$ & $n^{\max }$ \\
\hline 9 & 43 & 0.0101 & 0.0300 & 0.0038 & 119.5 & - & 1 & - \\
\hline 9 & 44 & 0.1106 & 0.3260 & 0.0417 & 119.5 & - & 1 & - \\
\hline 9 & 44 & 0.0688 & 0.3024 & 0.0453 & 162.9 & 8,787 & - & 3 \\
\hline 10 & 30 & 0.1579 & 0.3359 & 0.0411 & 96.4 & - & 1 & - \\
\hline 10 & 30 & 0.0693 & 0.3047 & 0.0457 & 162.9 & 8,849 & - & 3 \\
\hline 10 & 31 & 0.1389 & 0.3914 & 0.0551 & 119.5 & - & 2 & - \\
\hline 10 & 31 & 0.0866 & 0.3806 & 0.0571 & 162.9 & 10,876 & - & 3 \\
\hline 10 & 49 & 0.0489 & 0.1372 & 0.0192 & 119.5 & - & 1 & - \\
\hline 10 & 49 & 0.0303 & 0.1331 & 0.0200 & 162.9 & 4,270 & - & 3 \\
\hline 10 & 61 & 0.0315 & 0.1385 & 0.0208 & 162.9 & 4,413 & - & 3 \\
\hline 11 & 12 & 0.0000 & 0.0692 & 0.0000 & 200.0 & - & 2 & - \\
\hline 11 & 12 & 0.0000 & 0.0650 & 0.0000 & 200.0 & 3,132 & - & 3 \\
\hline 11 & 12 & 0.0000 & 0.0550 & 0.0000 & 300.0 & 3,767 & - & 3 \\
\hline 11 & 46 & 0.0549 & 0.1616 & 0.0206 & 119.5 & - & 1 & - \\
\hline 11 & 46 & 0.0340 & 0.1496 & 0.0224 & 162.9 & 4,709 & - & 3 \\
\hline 12 & 19 & 0.0111 & 0.0803 & 0.1762 & 429.4 & 12,760 & - & 3 \\
\hline 12 & 21 & 0.0097 & 0.0702 & 0.1546 & 429.4 & 11,346 & 2 & 3 \\
\hline 12 & 32 & 0.0101 & 0.0729 & 0.1605 & 429.4 & 11,733 & 2 & 3 \\
\hline 13 & 14 & 0.0515 & 0.1523 & 0.0192 & 119.5 & - & 1 & - \\
\hline 13 & 14 & 0.0319 & 0.1403 & 0.0210 & 162.9 & 4,461 & - & 3 \\
\hline 14 & 18 & 0.0872 & 0.2582 & 0.0326 & 119.5 & - & 1 & - \\
\hline 14 & 18 & 0.0541 & 0.2380 & 0.0357 & 162.9 & 7,067 & - & 3 \\
\hline 15 & 70 & 0.0225 & 0.1637 & 0.3661 & 429.4 & - & 2 & - \\
\hline 16 & 20 & 0.0753 & 0.2113 & 0.0297 & 119.5 & - & 2 & - \\
\hline 16 & 20 & 0.0467 & 0.2054 & 0.0308 & 162.9 & 6,198 & - & 3 \\
\hline 16 & 25 & 0.1166 & 0.1464 & 0.0156 & 67.1 & - & 1 & - \\
\hline 16 & 25 & 0.0283 & 0.1243 & 0.0186 & 162.9 & 4,033 & - & 3 \\
\hline 16 & 47 & 0.0272 & 0.1195 & 0.0179 & 162.9 & 3,906 & - & 3 \\
\hline 16 & 50 & 0.0382 & 0.1071 & 0.0150 & 119.5 & - & 2 & - \\
\hline 16 & 50 & 0.0236 & 0.1039 & 0.0156 & 162.9 & 3,489 & - & 3 \\
\hline 16 & 53 & 0.0775 & 0.3415 & 0.0513 & 162.9 & 9,844 & 1 & 3 \\
\hline 16 & 60 & 0.0263 & 0.1158 & 0.0173 & 162.9 & 3,805 & 1 & 3 \\
\hline 17 & 57 & 0.0354 & 0.1556 & 0.0233 & 162.9 & 4,868 & - & 3 \\
\hline 17 & 69 & 0.0352 & 0.1547 & 0.0232 & 162.9 & 4,845 & 1 & 3 \\
\hline 18 & 19 & 0.0000 & 0.0583 & 0.0000 & 200.0 & - & 2 & - \\
\hline 18 & 19 & 0.0000 & 0.0600 & 0.0000 & 200.0 & - & 1 & - \\
\hline 18 & 19 & 0.0000 & 0.0650 & 0.0000 & 200.0 & 3,132 & - & 3 \\
\hline 18 & 19 & 0.0000 & 0.0550 & 0.0000 & 300.0 & 3,767 & - & 3 \\
\hline 18 & 20 & 0.0071 & 0.0198 & 0.0028 & 119.5 & - & 2 & - \\
\hline 18 & 20 & 0.0044 & 0.0192 & 0.0029 & 162.9 & 1,230 & - & 3 \\
\hline 18 & 22 & 0.1365 & 0.3845 & 0.0541 & 119.5 & - & 1 & - \\
\hline 18 & 22 & 0.0851 & 0.3739 & 0.0560 & 162.9 & 10,695 & - & 3 \\
\hline
\end{tabular}

Table 4

Branch data of the modified northeastern

Thailand system (continued) 


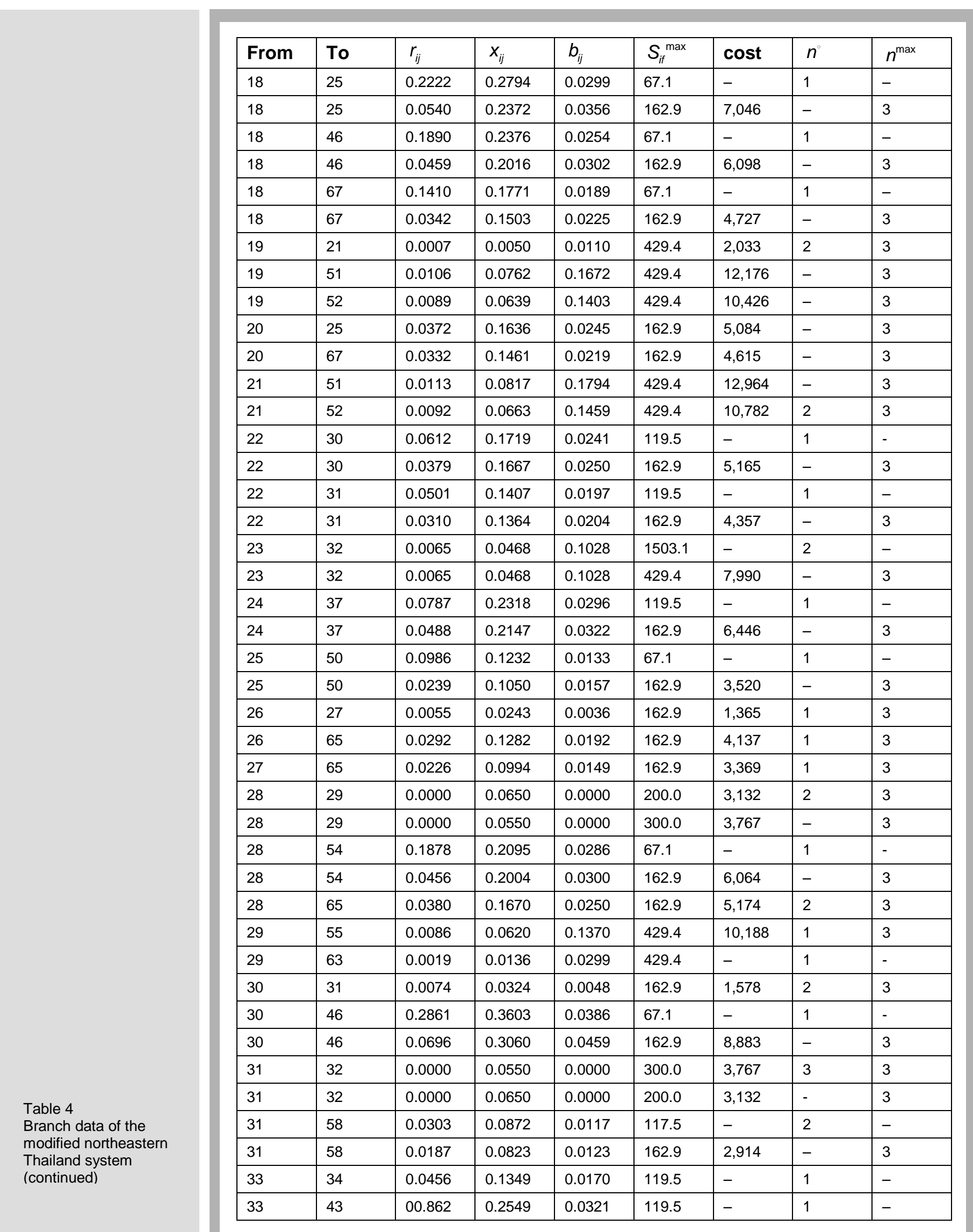




\begin{tabular}{|c|c|c|c|c|c|c|c|c|}
\hline From & To & $r_{i j}$ & $x_{i j}$ & $b_{i j}$ & $S_{\text {if }}^{\max }$ & cost & $n^{\circ}$ & $n^{\max }$ \\
\hline 34 & 48 & 0.0740 & 0.2080 & 0.0292 & 119.5 & - & 2 & - \\
\hline 34 & 48 & 0.1892 & 0.2378 & 0.0254 & 67.1 & - & 1 & - \\
\hline 35 & 36 & 0.0000 & 0.2338 & 0.0000 & 40.0 & - & 1 & - \\
\hline 36 & 44 & 0.0354 & 0.1557 & 0.0233 & 162.9 & 4,872 & - & 3 \\
\hline 36 & 53 & 0.0164 & 0.0721 & 0.0108 & 162.9 & 2,637 & 1 & 3 \\
\hline 36 & 54 & 0.0245 & 0.1078 & 0.0162 & 162.9 & 3,594 & - & 3 \\
\hline 36 & 60 & 0.0330 & 0.1452 & 0.0217 & 162.9 & 4,590 & 1 & 3 \\
\hline 37 & 71 & 0.0522 & 0.1534 & 0.0196 & 119.5 & - & 1 & - \\
\hline 37 & 71 & 0.0323 & 0.1420 & 0.0213 & 162.9 & 4,507 & - & 3 \\
\hline 37 & 72 & 0.0388 & 0.1707 & 0.0256 & 162.9 & 5,274 & 1 & 3 \\
\hline 38 & 44 & 0.0557 & 0.1656 & 0.0207 & 119.5 & - & 1 & - \\
\hline 38 & 44 & 0.0345 & 0.1518 & 0.0228 & 162.9 & 4,767 & - & 3 \\
\hline 38 & 71 & 0.0313 & 0.1374 & 0.0206 & 162.9 & 4,383 & - & 3 \\
\hline 38 & 72 & 0.0390 & 0.1159 & 0.0145 & 119.5 & - & 1 & - \\
\hline 38 & 72 & 0.0241 & 0.1061 & 0.0159 & 162.9 & 3,549 & - & 3 \\
\hline 39 & 48 & 0.0260 & 0.0731 & 0.0103 & 119.5 & - & 2 & - \\
\hline 39 & 64 & 0.0283 & 0.0355 & 0.0038 & 67.1 & - & 1 & - \\
\hline 39 & 71 & 0.0475 & 0.1332 & 0.0187 & 119.5 & - & 1 & - \\
\hline 39 & 71 & 0.1160 & 0.1456 & 0.0156 & 67.1 & - & 1 & - \\
\hline 39 & 71 & 0.0294 & 0.1292 & 0.0194 & 162.9 & 4,166 & - & 3 \\
\hline 39 & 72 & 0.0527 & 0.1480 & 0.0208 & 119.5 & - & 1 & - \\
\hline 39 & 72 & 0.0327 & 0.1436 & 0.0215 & 162.9 & 4,548 & - & 3 \\
\hline 40 & 58 & 0.0448 & 0.1290 & 0.0173 & 117.5 & - & 1 & - \\
\hline 40 & 58 & 0.0277 & 0.1219 & 0.0183 & 162.9 & 3,970 & - & 3 \\
\hline 41 & 59 & 0.0064 & 0.0283 & 0.0042 & 162.9 & 1,470 & - & 3 \\
\hline 41 & 69 & 0.0717 & 0.2013 & 0.0283 & 119.5 & 5,931 & 2 & 3 \\
\hline 42 & 59 & 0.0608 & 0.1789 & 0.0228 & 119.5 & - & 1 & - \\
\hline 42 & 73 & 0.0709 & 0.2087 & 0.0266 & 119.5 & - & 1 & - \\
\hline 44 & 53 & 0.0746 & 0.1608 & 0.0190 & 96.4 & - & 1 & - \\
\hline 44 & 53 & 0.0521 & 0.1535 & 0.0195 & 119.5 & - & 1 & - \\
\hline 44 & 53 & 0.0326 & 0.1434 & 0.0215 & 162.9 & 4,543 & - & 3 \\
\hline 44 & 54 & 0.0308 & 0.1354 & 0.0203 & 162.9 & 4,329 & - & 3 \\
\hline 44 & 72 & 0.1454 & 0.3139 & 0.0372 & 96.4 & - & 1 & - \\
\hline 44 & 72 & 0.0638 & 0.2803 & 0.0420 & 162.9 & 8,197 & - & 3 \\
\hline 45 & 50 & 0.0576 & 0.2532 & 0.0380 & 162.9 & 7,479 & 2 & 3 \\
\hline 47 & 50 & 0.0253 & 0.1110 & 0.0166 & 162.9 & 3,678 & 1 & 3 \\
\hline 47 & 60 & 0.0317 & 0.1392 & 0.0209 & 162.9 & 4,432 & - & 3 \\
\hline 48 & 64 & 0.0467 & 0.0586 & 0.0063 & 67.1 & - & 1 & - \\
\hline 50 & 51 & 0.0000 & 0.0675 & 0.0000 & 200.0 & - & 1 & - \\
\hline 50 & 51 & 0.0000 & 0.0675 & 0.0000 & 200.0 & - & 1 & - \\
\hline 50 & 51 & 0.0000 & 0.0650 & 0.0000 & 200.0 & 3,132 & 1 & 3 \\
\hline 50 & 51 & 0.0000 & 0.0550 & 0.0000 & 300.0 & 3,767 & - & 3 \\
\hline 50 & 74 & 0.0701 & 0.1970 & 0.0276 & 119.5 & - & 2 & - \\
\hline 50 & 74 & 0.0435 & 0.1912 & 0.0287 & 162.9 & 5,819 & - & 3 \\
\hline
\end{tabular}

Table 4

Branch data of the modified northeastern Thailand system (continued) 


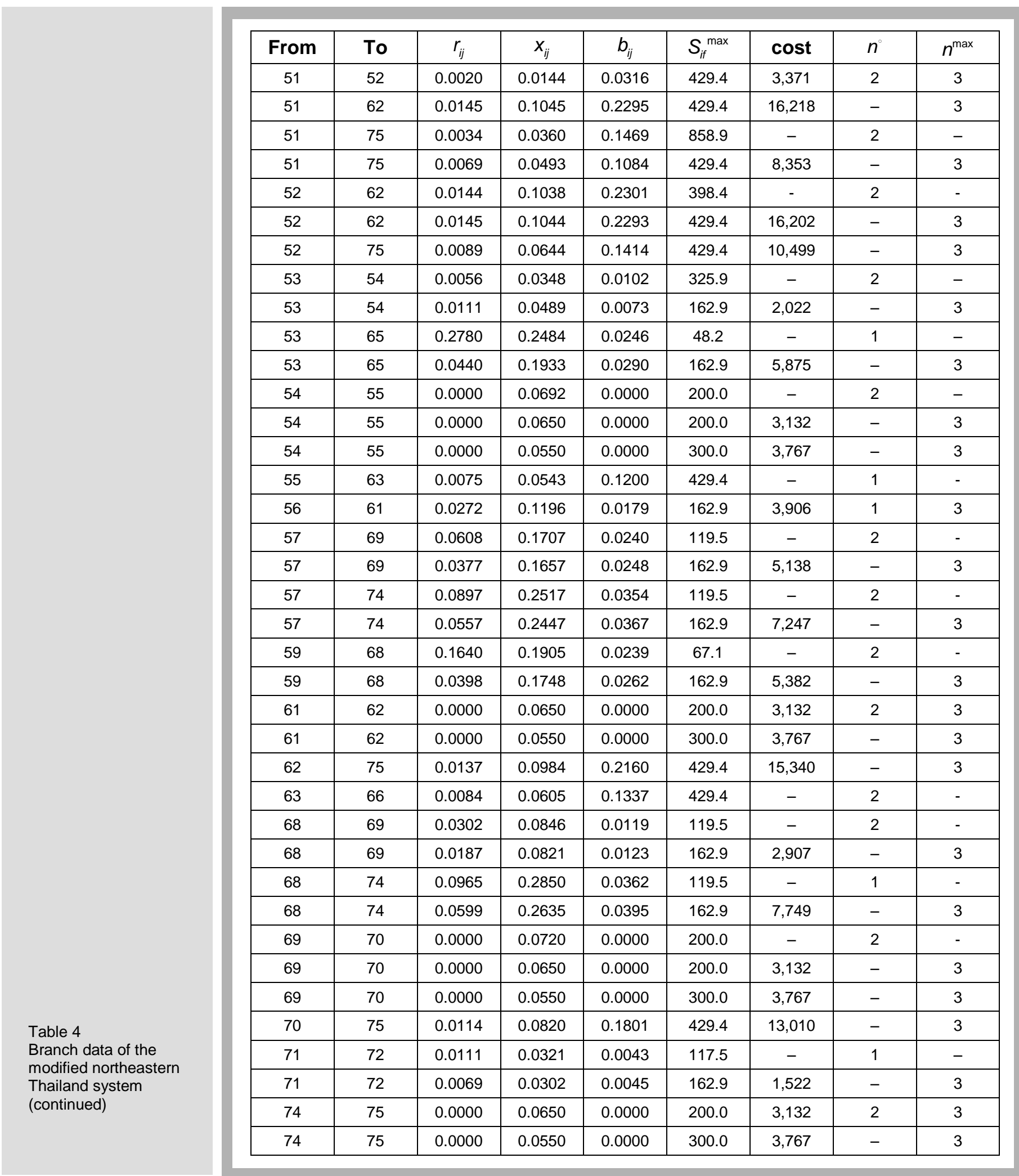




\section{REFERENCES}

[1] G. Latorre, R. D. Cruz, J. M. Areiza, and A. Villegas, "Classification of publication and models on transmission expansion planning," IEEE Transaction on Power Systems, vol. 18, no. 2, pp. 938-946, 2003.

[2] R. Romero, A. Monticelli, A. Garcia, and S. Haffner, "Test systems and mathematical models for transmission network expansion planning," IEE Proceedings - Generation, Transmission and Distribution, vol. 149, no. 1, pp. 27-36, 2002.

[3] R. Romero, C. Rocha, J. Mantovani, and I. Sanchez, "Constructive heuristic algorithm for the DC model in network transmission expansion planning," IEE Proceedings - Generation, Transmission and Distribution, vol. 152, no. 2, pp. 277-282, 2005.

[4] M. V. F. Pereira and L. M. V. G. Pinto, "Application of sensitivity analysis of local supplying capability to interactive transmission expansion," IEEE Transaction on Power Systems, vol. PAS-104, no. 2, pp. 381-389, 1985.

[5] E. J. de Oliveira, J. Ivo Chaves da Silva, J. L. R. Pereira, and J. Sandoval Carneiro, "Transmission system expansion planning using a sigmoid function to handle integer investment variables," IEEE Transaction on Power Systems, vol. 20, no. 3, pp. 1616-1621, 2005.

[6] Z. Michalewicz and D. B.Fogel, How to Solve it: Modern Heuristics. Springer-Verlag Berlin Heidelberg, 2000.

[7] M. Fischetti and A. Lodi, "Local branching," Mathematical Programming, Series B, vol. 98, pp. 23-47, 2003.

[8] E. L. da Silva, H. A. Gil, and J. M. Areiza, "Transmission network expansion planning under an improved genetic algorithm," IEEE Transaction on Power Systems, vol. 15, no. 3, pp. 1168-1175, 2000.

[9] R. A. Gallego, R. Romero, and A. J. Monticelli, "Tabu search algorithm for network synthesis," IEEE Transaction on Power Systems, vol. 15, no. 2, pp. 490-495, 2000 .

[10] I. Sanchez, R. Romero, J. Mantovani, and M. Rider, "Transmission expansion planning using the DC model and nonlinear-programming technique," IEE Proceedings - Generation, Transmission and Distribution, vol. 152, no. 6, pp. 763-769, 2005.

[11] S. Haffner, A. Monticelli, A. Garcia, A. Mantovani, and R. Romero, "Branch and bound algorithm for transmission system expansion planning using a transportation model," IEE Proceedings - Generation, Transmission and Distribution, vol. 147, no. 3, pp. 149-156, 2000.

[12] L. Bahiense, G. C. Oliveira, M. V. F. Pereira, and S. Granville, "A mixed integer disjunctive model for transmission network expansion," IEEE Transaction on Power Systems, vol. 16, no. 3, pp. 560-565, 2001.

[13] S. Binato, M. V. F. Pereira, and S. Granville, "A new Benders decomposition approach to solve power transmission network design problems," IEEE Transaction on Power Systems, vol. 16, no. 2, pp. 235-240, 2001.

[14] A. H. Escobar, R. A. Gallego, and R. Romero, "Multistage and coordinated planning of the expansion of transmission systems," IEEE Transaction on Power Systems, vol. 19, no. 2, pp. 735-744, 2004.

[15] R. Romero, C. Rocha, M. Mantovani, and J. Mantovani, "Analysis of heuristic algorithms for the transportation model in static and multistage planning in network expansion systems," IEE Proceedings - Generation, Transmission and Distribution, vol. 150, no. 5, pp. 521526, 2003.

[16] R. Fang and D. J. Hill, "A new strategy for transmission expansion in competitive electricity markets," IEEE Transaction on Power Systems, vol. 18, no. 1, pp. 374-380, 2003. 